\title{
Glucosamine Induces Insulin Resistance In Vivo by Affecting GLUT 4 Translocation in Skeletal Muscle Implications for Glucose Toxicity
}

\author{
Alain D. Baron, ${ }^{*}$ Jin-Su Zhu, ${ }^{*}$ Ju-Hong Zhu, ${ }^{*}$ Heather Weldon, ${ }^{*}$ Lidia Maianu, ${ }^{\star}$ and W. Timothy Garvey \\ *Department of Medicine, Indiana University School of Medicine, and the Richard L. Roudebush Veterans Affairs Medical Center, \\ Indianapolis, Indiana 46202; and ${ }^{\ddagger}$ Department of Medicine, Medical University of South Carolina, and the Ralph H. Johnson \\ Veterans Affairs Medical Center, Charleston, South Carolina 29425
}

\begin{abstract}
Glucosamine (GImn), a product of glucose metabolism via the hexosamine pathway, causes insulin resistance in isolated adipocytes by impairing insulin-induced GLUT 4 glucose transporter translocation to the plasma membrane. We hypothesized that GImn causes insulin resistance in vivo by a similar mechanism in skeletal muscle. We performed euglycemic hyperinsulinemic clamps $\left(12 \mathrm{mU} / \mathrm{kg} / \mathrm{min}+{ }^{3} \mathrm{H}-\right.$ 3-glucose) in awake male Sprague-Dawley rats with and without GImn infusion at rates ranging from 0.1 to $6.5 \mathrm{mg}$ / $\mathrm{kg} / \mathrm{min}$. After $4 \mathrm{~h}$ of euglycemic clamping, hindquarter muscles were quick-frozen and homogenized, and membranes were subfractionated by differential centrifugation and separated on a discontinuous sucrose gradient $(25,30$, and $35 \%$ sucrose). Membrane proteins were solubilized and immunoblotted for GLUT 4. With GImn, glucose uptake (GU) was maximally reduced by $33 \pm 1 \%, P<0.001$. The apparent GImn dose to reduce maximal GU by $50 \%$ was $0.1 \mathrm{mg} / \mathrm{kg} /$ min or 1/70th the rate of GU on a molar basis. Control galactosamine and mannosamine infusions had no effect on GU. Relative to baseline, insulin caused a 2.6-fold increase in GLUT 4 in the $25 \%$ membrane fraction (f), $P<0.01$, and a $40 \%$ reduction in the $35 \%$ f, $P<0.05$, but had no effect on GLUT 4 in the 30\%f, $P=$ NS. Addition of Glmn to insulin caused a $41 \%$ reduction of GLUT 4 in the $25 \%$, $P<0.05$, a $29 \%$ fall in the $30 \%$ f, and prevented the reduction of GLUT 4 in the 35\%f. The $30 \%$ membranes were subjected to a second separation with a 27 and $30 \%$ sucrose gradient. Insulin mobilized GLUT 4 away from the $30 \%$ f, $P<0.05$, but not the $27 \%$ f. In contrast, GImn reduced GLUT 4 in the $27 \%$ f, $P<0.05$, but not the $30 \%$ f. Thus, GImn appears to alter translocation of an insulin-insensitive GLUT 4 pool. Coinfusion of GImn did not alter enrichment of the sarcolemmal markers $5^{\prime}$-nucleotidase, $\mathrm{Na}^{+}$I $\mathrm{K}^{+} \mathrm{ATPase}$, and phospholemman in either 25,30 , or $35 \% \mathrm{f}$. Thus, GImn completely blocked movement of GLUT 4 induced by insulin. GImn is a potent inducer of insulin resis-
\end{abstract}

Address correspondence to Alain Baron, M.D., Division of Endocrinology and Metabolism, Indiana University School of Medicine, 541 North Clinical Drive, Clinical Building 459, Indianapolis, IN 46202-5111. Phone: 317-274-1339; FAX: 317-278-0658; E-mail: alainb@medicine.dmed.iupui.edu

Received for publication 3 March 1995 and accepted in revised form 1 September 1995.

The Journal of Clinical Investigation, Inc.

Volume 96, December 1995, 2792-2801 tance in vivo by causing (at least in part) a defect intrinsic to GLUT 4 translocation and/or trafficking. These data support a potential role for GImn to cause glucose-induced insulin resistance (glucose toxicity). (J. Clin. Invest. 1995. 96:2792-2801.) Key words: insulin resistance - glucose transporter • GLUT 4 - euglycemic clamp • hexosamine

\section{Introduction}

Insulin resistance is a major contributing factor in the pathogenesis of non-insulin-dependent diabetes mellitus (NIDDM) ${ }^{1}$ (1) and is characterized by reduced rates of insulin-mediated glucose uptake into skeletal muscle (2). It is now clear that both inherited and acquired forms of insulin resistance exist. Among the more intriguing mechanisms of acquired forms of insulin resistance is glucose-induced desensitization or glucose toxicity $(1-4)$. In vivo data have suggested that hyperglycemia per se can induce or worsen insulin resistance. In patients with NIDDM, induction of euglycemia by weight reduction (5), sulfonylureas $(6,7)$, or insulin therapy $(8,9)$ results in significant amelioration of insulin sensitivity, as well as increased glucose transport rates measured in adipocytes $(5,10)$ and skeletal muscle (11) obtained from these patients. Likewise, patients with insulin-dependent diabetes mellitus (IDDM) in poor glycemic control exhibit insulin resistance which can be reversed by intensified insulin therapy designed to achieve near normal glycemia $(12,13)$. Rats made diabetic by subtotal pancreatectomy or streptozotocin exhibit marked impairment in insulin's action to stimulate glucose uptake in muscle and fat which can be reversed by induction of euglycemia by exogenous insulin or by promotion of glycosuria with phlorizin (14-16). Finally, there are multiple in vitro studies that demonstrate direct effects of glucose to impair insulin-stimulated glucose transport in perfused target tissues (17) and cultured cell systems (2). Thus, there is a large body of data supporting the concept that glucose per se can induce desensitization of insulin's action to stimulate glucose uptake.

To study underlying mechanisms, we have previously used the primary cultured adipocyte system to study the long-term ability of glucose to regulate the glucose transport system in a classic insulin target cell (18-21). In these cells, we found that, with chronic exposure, glucose and insulin acted synergistically to reduce the activity of the glucose transport system.

1. Abbreviations used in this paper: GFAT, glutamine:fructose-6- $P$ amidotransferase; HGO, hepatic glucose output; IDDM, insulin-dependent diabetes mellitus; NIDDM, non-insulin-dependent diabetes mellitus; $R_{\mathrm{a}}$, rate of glucose appearance. 
Further studies revealed that glucose-induced insulin resistance arose via a novel mechanism which involved impaired recruitment of intracellular glucose transporters to the cell surface, without a change in the total cellular number of transporters $(18,19)$. The ability of glucose to regulate its own uptake was dependent on its intracellular metabolism. Of interest, we found that glucosamine, a product of glucose metabolism, could substitute for glucose and was more potent than glucose in promoting desensitization of the glucose transport system (18).

Following these initial observations, Marshall et al. conducted an elegant series of experiments implicating the hexosamine pathway of glucose metabolism in substrate-induced downregulation of the adipocyte glucose transport system (22$26)$. Glucose enters this multistep pathway at fructose-6- $P$ with $N$-acetylglucosamine-6- $P$ as the major end product. $N$-acetylglucosamine-6- $P$ ultimately becomes incorporated into macromolecules such as gangliosides, glycolipids, glycoproteins, and proteoglycans. The first and rate-limiting enzyme for this pathway is glutamine: fructose-6- $P$ amidotransferase (GFAT), which uses glutamine as an amide donor to convert fructose-6$P$ to glucosamine-6- $P$. Involvement of hexosamine pathway products in glucose-induced insulin resistance is supported by three observations: ( $a$ ) glutamine is a necessary cofactor, along with glucose and insulin, for desensitization of the glucose transport system; $(b)$ azaserine, a glutamine antagonist which irreversibly inhibits glutamine amidotransferases (i.e., GFAT), specifically blocks the ability of glucose, insulin, and glutamine to regulate glucose transport; and (c) glucosamine, which enters the hexosamine pathway distal to GFAT, can alone impair the glucose transport system, and thus accounts for its enhanced potency relative to glucose.

The purpose of the current study was to examine whether the hexosamine pathway plays a role in the induction of insulin resistance in vivo, and if so by what mechanism. Because glucose uptake occurs principally in skeletal muscle, we specifically examined whether the glucose transporter translocation defect observed in adipocytes in vitro was also displayed in skeletal muscle in vivo. In our experiments, we infused rats with glucosamine and assessed effects on in vivo insulin action using the euglycemic hyperinsulinemic clamp technique. We also examined whether glucosamine altered the subcellular distribution and translocation of GLUT 4 in skeletal muscle after insulin stimulation. These studies have established a new animal model for glucose toxicity. Our observations are directly relevant to the pathogenesis of insulin resistance in NIDDM since they provide an explanation of how hyperglycemia in vivo could lead to insulin resistance at the cellular level in skeletal muscle.

\section{Methods}

Protocol for in vivo studies. Male Sprague-Dawley rats (Harlan, Indianapolis, IN) weighing between 250 and 350 grams (Tables I-III) were studied while awake and unstressed after overnight food deprivation. 3-4 $\mathrm{d}$ before the study, each rat had undergone surgery for implantation of both carotid artery and jugular vein catheters (see below). On the day of the study, each rat had fully recovered as evidenced by progressive weight gain which at minimum led to the recovery of presurgical weight. On the morning of the study, the arterial and venous catheters were connected to withdrawal and infusion syringes, respectively.

Study I. To examine the dose-response effect of glucosamine infusion on rates of insulin-mediated glucose uptake, five groups of rats ( $n$ $=5$ in each group, Table I) underwent a euglycemic clamp (see methods below) performed at a single and maximally effective insulin infusion rate of $12 \mathrm{mU} / \mathrm{kg} / \mathrm{min}$ (27). After obtaining basal bloods, each clamp was carried out for $120 \mathrm{~min}$ to achieve steady state rates of glucose disposal. After $120 \mathrm{~min}$, each group received a glucosamine infusion at rates of $0.1,0.8,1.7,3.5$, or $6.5 \mathrm{mg} / \mathrm{kg} / \mathrm{min}$ which was continued for an additional $120 \mathrm{~min}$. Thus, with this design, each rat served as its own control and each clamp study lasted a total of $4 \mathrm{~h}$. Glucosamine infusate concentrations were adjusted so as to infuse the same volume in each experimental condition.

Study II. Because of the long duration of the clamp in study I (4 h), it was important to control for the effect of time on measured parameters. To this end, we performed euglycemic hyperinsulinemic clamps at an insulin infusion rate of $12 \mathrm{mU} / \mathrm{kg} / \mathrm{min}$. Beginning at time 0 of the clamp, separate groups of rats ( $n=5$ each, Table II) were concomitantly infused with either saline (continued for $200 \mathrm{~min}$ ) or glucosamine (continued for $120 \mathrm{~min}$ ). Glucosamine was infused at a dose of $3.5 \mathrm{mg} / \mathrm{kg} / \mathrm{min}$ which was determined in study I to result in maximal reductions of insulin-mediated glucose uptake. Fluid volumes delivered during the clamps were matched in both groups.

Study III. To examine the specificity of glucosamine to cause insulin resistance, separate groups of rats ( $n=4$ each, Table III) underwent euglycemic hyperinsulinemic clamps in a similar design to study II with the infusion of glucosamine, mannosamine, or galactosamine at doses of $3.5 \mathrm{mg} / \mathrm{kg} / \mathrm{min}$. Animals studied during euglycemic hyperinsulinemic clamps without glucosamine (saline) in study II served as the saline control group in study III.

Methods for glucose clamp in awake chronically catheterized rats. Catheterizations were performed under ketamine hydrochloride anesthesia ( $10 \mathrm{mg} / 100$-gram animal, given intramuscularly; Fort Dodge Laboratories, Inc., Fort Dodge, IA). Polyethylene tubing (internal diameter 0.23 in $\times$ outer diameter 0.038 in; Becton Dickinson, Parsippany, NJ) was inserted into the left carotid artery and silastic tubing (internal diameter 0.20 in $\times$ outer diameter 0.037 in; Dow Corning, Midland, MI) was inserted into the right jugular vein. Both catheters were tunneled under the skin and exteriorized in the nuchal area. Animals were allowed to recover over the following 3-4 d during which time normal behavior and progressive weight gain were observed. Catheters were kept patent by flushing with a heparin solution on alternate days. Except during flushing and during infusion studies, catheters were kept capped at all times.

Euglycemic hyperinsulinemic clamp studies were performed after $16 \mathrm{~h}$ of overnight fast using a modification of the method described by Smith et al. (27). Arterial and venous lines were connected to sampling and infusion syringes, respectively. Glucose turnover in both the basal and insulin-stimulated state was assessed isotopically by bolus injection of $6 \mu \mathrm{Ci}{ }^{3} \mathrm{H}$-3-glucose (New England Nuclear, Boston, MA) followed by a constant infusion at $0.1 \mu \mathrm{Ci} / \mathrm{min}$. Blood for measurement of basal glucose specific activity was obtained after $60 \mathrm{~min}$ of constant tracer infusion at time $-20,-10$, and $0 \mathrm{~min}$. Blood for the measurement of basal insulin concentrations was obtained at time -20 and $0 \mathrm{~min}$. Insulin infusion (Humulin-R; Eli Lilly and Co., Indianapolis, IN) was then initiated at time 0 of the clamp at a rate of $12 \mathrm{mU} / \mathrm{kg} / \mathrm{min}$. The insulin infusate was mixed in a heparinized $(9 \mathrm{U} / \mathrm{ml}$ heparin $)$ solution of glucose tracer and blood (obtained from cardiac puncture of a littermate) drawn up into a 5-ml syringe and delivered continuously via a compact Harvard pump (model 975; Harvard Apparatus Inc., South Natick, MA). Serum glucose concentration was measured at 5-10-min intervals and clamped at euglycemia with a variable infusion of $20 \%$ dextrose solution (Abbott Laboratories, North Chicago, IL) via an adjustable Razel pump (model A-99; Razel Scientific Instruments, Stamford, CT). Clamps were carried out for 2 (study II), 3 (study II, saline only), or $4 \mathrm{~h}$ (studies I and II). Serum insulin levels were measured at time 100 and $120 \mathrm{~min}$ (studies I and II), at time 180 and $200 \mathrm{~min}$ (study II, saline only), and at time 230 and $240 \mathrm{~min}$ ( studies I and III) during hexosamine infusion. Blood samples were obtained at time 100, 110, and 120 min to measure steady state glucose specific activity during euglycemic hyperinsulinemia (studies I, II, and III) and at time 220, 230, and 
Table I. Characteristics of Animals in Study I

\begin{tabular}{lccccc}
\hline Glucosamine dose (mg/kg/min) & 0.1 & 0.8 & 1.7 & 3.5 & \multicolumn{1}{c}{5.5} \\
$n$ & 5 & 5 & 5 & 5 & $279 \pm 15$ \\
Wt (grams) & $258 \pm 5$ & $275 \pm 16$ & $277 \pm 14$ & $259 \pm 5$ & $8.5 \pm 3.5$ \\
Fasting insulin $(\mu \mathrm{U} / \mathrm{ml})$ & $6.1 \pm 2.4$ & $7.5 \pm 3.8$ & $6.2 \pm 2.9$ & $3.8 \pm 1.1$ & $121 \pm 4$ \\
Fasting glucose $(\mathrm{mg} / \mathrm{dl})$ & $116 \pm 5$ & $119 \pm 3$ & $110 \pm 4$ & $124 \pm 8$ & $8.5 \pm 0.7$ \\
Basal HGO $(\mathrm{mg} / \mathrm{kg} / \mathrm{min})$ & $8.6 \pm 2.6$ & $7.0 \pm 0.7$ & $8.0 \pm 1.0$ & $11.6 \pm 1.4$ & $268 \pm 52$ \\
Clamp insulin - saline $(\mu \mathrm{U} / \mathrm{ml})$ & $225 \pm 50$ & $240 \pm 68$ & $221 \pm 32$ & $229 \pm 89$ & $117 \pm 6$ \\
Clamp glucose - saline $(\mathrm{mg} / \mathrm{dl})$ & $111 \pm 3$ & $112.8 \pm 3$ & $117 \pm 1$ & $111 \pm 4$ & $311 \pm 39$ \\
Clamp insulin - glucosamine $(\mu \mathrm{U} / \mathrm{ml})$ & $279 \pm 32$ & $305 \pm 27$ & $282 \pm 71$ & $277 \pm 47$ & $122 \pm 6$ \\
Clamp glucose - glucosamine $(\mathrm{mg} / \mathrm{dl})$ & $117 \pm 4$ & $117 \pm 3$ & $112 \pm 5$ & $121 \pm 11$ & \\
\end{tabular}

240 min during hexosamine infusion (studies I and III). Under basal conditions and during insulin infusion, the rate of glucose appearance $\left(R_{\mathrm{a}}\right)$ was calculated by dividing the known infusion rate of tritium counts by the glucose specific activity. The rate of hepatic glucose output (HGO) was calculated as $R_{\mathrm{a}}$ minus the glucose infusion rate (HGO $\left.=R_{\mathrm{a}}-\mathrm{GINF}\right)$. Under steady state conditions $R_{\mathrm{a}}$ is equal to the rate of glucose disappearance $\left(R_{\mathrm{d}}\right)$.

Membrane subfractionation of skeletal muscle. To study the role of GLUT 4 glucose transporter translocation on glucosamine-induced insulin resistance, separate groups of rats were studied under basal conditions and as per the protocol used in study I. Basal muscles were obtained from chronically catheterized animals at the end of a 4-h infusion of saline. All other animals underwent a euglycemic hyperinsulinemic clamp ( $12 \mathrm{mU} / \mathrm{kg} / \mathrm{min})$ which lasted $4 \mathrm{~h}$. During the initial $120 \mathrm{~min}$, only insulin, glucose, and saline were infused. During the final $120 \mathrm{~min}$, one group received a glucosamine infusion $(3.5 \mathrm{mg} / \mathrm{kg} / \mathrm{min})$. At the end of the 4-h clamp, all animals were killed for extirpation of skeletal muscle. Hindlimb muscle was rapidly dissected free of connective tissue. Gastrocnemius, soleus, and vastus muscle groups were quick-frozen in liquid nitrogen and stored at $-80^{\circ} \mathrm{C}$ for later study. Muscles from individual rats were placed in Tris buffer $(5 \mathrm{ml}$ for every 1 gram of tissue), $\mathrm{pH} 7.4$, at $4^{\circ} \mathrm{C}$, containing $20 \mathrm{mM}$ Tris-base, $0.25 \mathrm{M}$ sucrose, $0.1 \mathrm{mM}$ EDTA, and protease inhibitors including $5 \mu \mathrm{g} / \mathrm{ml}$ leupeptin, 5 $\mu \mathrm{g} / \mathrm{ml}$ aprotinin, $1 \mu \mathrm{g} / \mathrm{ml}$ pepstatin, and $400 \mu \mathrm{M}$ phenylmethanesulfonyl fluoride. All steps were performed at $4^{\circ} \mathrm{C}$. Methods used for membrane subfractionation were those described by Klip and colleagues $(28,29)$ with minor modifications. Briefly, muscle tissue was homogenized first with a Polytron tissue disrupter (Brinkmann, Westbury, NY) using five 5 -s bursts at a setting of 5 and then with 10 up-and-down strokes of a motor-driven Teflon pestle in a glass homogenization tube (Thomas, Philadelphia, PA). The homogenate was centrifuged at $1,000 \mathrm{~g}$ for 10 $\mathrm{min}$, and the supernatant was saved. The resulting pellet was resuspended in the buffer, rehomogenized with the glass homogenization

Table II. Characteristics of Animals in Study II

\begin{tabular}{lcc}
\hline & \begin{tabular}{c}
$12 \mathrm{mU} / \mathrm{kg} / \mathrm{min}+$ \\
$50 \mathrm{mg}$ glucosamine \\
\multicolumn{1}{c}{ Study }
\end{tabular} & $\begin{array}{c}12 \mathrm{mU} / \mathrm{kg} / \mathrm{min}+ \\
\text { saline } \times 4 \mathrm{~h}\end{array}$ \\
\hline$n$ & $5.5 \mathrm{mg} / \mathrm{kg} / \mathrm{min} \times 2 \mathrm{~h}$ & 5 \\
Wt (grams) & $308 \pm 17$ & $268 \pm 19$ \\
Fasting insulin $(\mu \mathrm{U} / \mathrm{ml})$ & $18.2 \pm 6.9$ & $12.6 \pm 6.0$ \\
Fasting glucose $(\mathrm{mg} / \mathrm{dl})$ & $120 \pm 2$ & $126 \pm 2$ \\
Basal HGO $(\mathrm{mg} / \mathrm{kg} / \mathrm{min})$ & $6.7 \pm 0.6$ & $6.2 \pm 0.3$ \\
Clamp insulin $(\mu \mathrm{U} / \mathrm{ml})$ & $248 \pm 51$ & $311 \pm 94$ \\
Clamp glucose $(\mathrm{mg} / \mathrm{dl})$ & $122 \pm 5$ & $117 \pm 3$
\end{tabular}

tube and Teflon pestle as described above, and recentrifuged at 1,000 $g$ for $10 \mathrm{~min}$. The resulting pellet was discarded, and the supernatant was combined with the first supernatant and centrifuged at $9,000 \mathrm{~g}$ for $10 \mathrm{~min}$. The resulting supernatant was then centrifuged at $190,000 \mathrm{~g}$ for $60 \mathrm{~min}$; the resuspended pellet constituted the total postnuclear membrane fraction. Total membranes were then applied to a discontinuous sucrose gradient containing 25,30 , and $35 \%$ sucrose (wt/vol) solutions, and centrifuged at $40,000 \mathrm{~g}$ for $16 \mathrm{~h}$ in a swinging bucket rotor. Membranes were collected at the interface of each of the sucrose gradients, resuspended in the Tris buffer, pelleted by centrifugation at 190,000 $g$ for $60 \mathrm{~min}$, and resuspended in Tris buffer. Membranes from the 30\% sucrose fraction were also subjected to a second discontinuous sucrose density gradient containing 27 and $30 \%$ sucrose ( $w t / v o l$ ) solutions. The second gradient was centrifuged at $40,000 \mathrm{~g}$ for $3.5 \mathrm{~h}$ in a swinging bucket rotor. Membranes were collected at the interface of the 27 and $30 \%$ sucrose solutions in the Tris buffer, pelleted at $190,000 \mathrm{~g}$ for 60 min, and resuspended in Tris buffer. All suspensions of membrane subfractions were kept at $-80^{\circ} \mathrm{C}$ for subsequent immunoblot analyses. To monitor membrane subfractionation, we measured 5 '-nucleotidase activity using the method of Avruch and Wallach (30), oubain-inhibitable $\mathrm{Na}^{+} / \mathrm{K}^{+}$ATPase activity as described previously (31), and immunoreactive phospholemman $(32,33)$ (see below) as markers for sarcolemma in total membranes and membrane subfractions.

Immunoblot analyses. 30- $\mu \mathrm{g}$ membrane proteins were solubilized in Laemmli sample buffer (34) and resolved by SDS-PAGE on 1.5$\mathrm{mm}$ slab gels. The proteins were then electrophoretically transferred to nitrocellulose filters (35). Immunological detection of GLUT 4 glucose transporters was accomplished as described previously (36). Nitrocellu-

Table III. Characteristics of Animals in Study III

\begin{tabular}{|c|c|c|c|c|}
\hline $\begin{array}{c}\text { Dose } \\
3.5 \mathrm{mg} / \mathrm{kg} / \mathrm{min}\end{array}$ & Saline & Glucosamine & Galactosamine & Mannosamine \\
\hline$n$ & 5 & 4 & 4 & 4 \\
\hline Wt (grams) & $268 \pm 19$ & $310 \pm 21$ & $277 \pm 16$ & $301 \pm 6$ \\
\hline $\begin{array}{l}\text { Fasting insulin } \\
\qquad(\mu \mathrm{U} / \mathrm{ml})\end{array}$ & $12.6 \pm 6.0$ & $18.2 \pm 6.8$ & $14.4 \pm 5.2$ & $11.7 \pm 3.3$ \\
\hline $\begin{array}{l}\text { Fasting glucose } \\
\qquad(\mathrm{mg} / \mathrm{dl})\end{array}$ & $126 \pm 2$ & $121 \pm 2.5$ & $119 \pm 205$ & $117 \pm 3.1$ \\
\hline $\begin{array}{l}\text { Basal HGO } \\
(\mathrm{mg} / \mathrm{kg} / \mathrm{min})\end{array}$ & $6.2 \pm 0.3$ & $6.5 \pm 0.7$ & $6.4 \pm 0.5$ & $6.5 \pm 0.9$ \\
\hline $\begin{array}{l}\text { Clamp insulin } \\
(\mu \mathrm{U} / \mathrm{ml})\end{array}$ & $311 \pm 94$ & $223 \pm 44$ & $275 \pm 62$ & $263 \pm 57$ \\
\hline $\begin{array}{l}\text { Clamp glucose } \\
\text { (mg/dl) }\end{array}$ & $117 \pm 3.0$ & $116 \pm 2.7$ & $115 \pm 4.9$ & $123 \pm 3.7$ \\
\hline
\end{tabular}



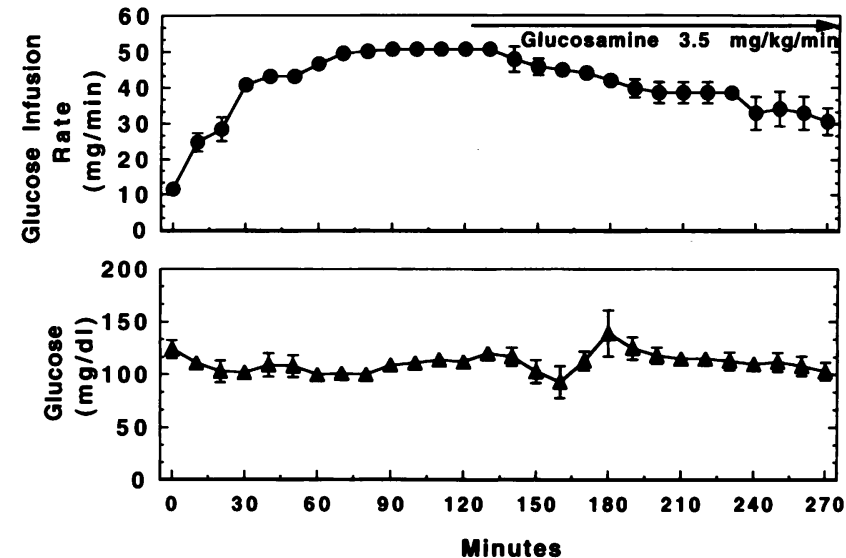

Figure 1. Effect of glucosamine on glucose infusion rates and serum glucose concentrations during a representative euglycemic hyperinsulinemic clamp study. Awake chronically catheterized rats were infused with insulin ( $12 \mathrm{mU} / \mathrm{kg} / \mathrm{min}$ ) for $4 \mathrm{~h}$. During the first $2 \mathrm{~h}$, each animal was coinfused with saline only; subsequently, animals received an infusion of glucosamine at various doses $(0.1,0.8,1.7,3.5$, and $6.5 \mathrm{mg} /$ $\mathrm{kg} / \mathrm{min}$ ). Five animals were studied at each dose. Thus, each animal served as its own control to assess the effect of glucosamine dose on insulin's action to reduce rates of glucose uptake. In the above example, five rats were studied during a euglycemic hyperinsulinemic clamp. At $120 \mathrm{~min}$, a glucosamine infusion $(3.5 \mathrm{mg} / \mathrm{kg} / \mathrm{min})$ was begun. Glucose infusion rates required to maintain euglycemia are shown. Serum glucose concentrations were kept near the basal level throughout. Data are mean \pm SEM

lose filters (Western blots) were incubated with rabbit antiserum ( 1:1,000 dilution) specific for the $\mathrm{COOH}$-terminal segment ( 12 amino acids) of rat GLUT 4 (37) (East Acres Biologicals, Southbridge, MA; catalog number RaIRGT) followed by ${ }^{125}$ I-protein A. Detection of phospholemman was accomplished by incubating filters with affinity-purified rabbit antiserum (1:5,000 dilution) raised against canine phospholemman $(32,33)$ (kind gift of Dr. Larry Jones, Indiana University) followed by ${ }^{125}$ I-protein A. GLUT 4 migrated as a relatively broad band and so, for quantitation of GLUT 4 levels, the total band was excised for measurement of radioactive counts, whereas scanning densitometry (Bio-Rad, Richmond, CA) of autoradiographs was used to measure phospholemman which migrated as a sharp-focused band. In either instance, background measurements were subtracted from signal to obtain relative levels of the immunoreactive protein, and measurements were consistently in the range where the relationship between increasing amounts of membrane protein and signal was linear (data not shown).

Other assays and statistics. During the clamp study, plasma glucose concentration was measured by the glucose oxidase method using a glucose analyzer (model 23A; Yellow Springs Instruments, Yellow Springs, $\mathrm{OH}$ ). Glucose specific activity was determined in plasma deproteinized with $0.6 \mathrm{~N}$ perchloric acid; duplicate samples of the supernatant were evaporated, resuspended in deionized water and liquid scintillation fluid (Ecoscint; National Diagnostics, Manville, $\mathrm{NJ}$ ), and counted. Results were corrected for recovery of radioactive counts and counting efficiency. Recovery was determined by counting a serum sample spiked with a known amount of radioactive counts. Serum insulin concentrations were measured by a double-antibody radioimmunoassay (Linco, St. Louis, MO) using rat insulin antibody to measure fasting insulin levels and human insulin antibody to measure circulating immunoreactive human insulin during the clamp studies. In muscle membranes, protein was measured using the method of Bradford (38) with crystalline BSA serving as the standard.

All data are reported as mean \pm SEM. Comparisons were performed by ANOVA using the Statview 4.0 software package (Abacus Concepts,

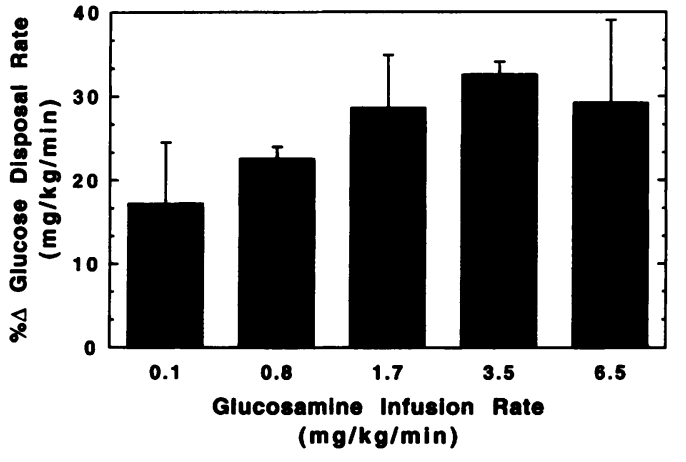

Figure 2. Effect of various doses of glucosamine on glucose disposal rates during euglycemic hyperinsulinemic clamp studies. Shown above are the relative reductions in glucose disposal rates (isotopically determined rates of glucose uptake) calculated in each rat as the percent reduction in glucose disposal rate during saline versus glucosamine infusion (as illustrated in Fig. 1). Each bar represents the mean \pm SEM of five studies.

Inc., Berkeley, CA) followed by a Fisher protected least significant difference test. Significance was accepted at $P<0.05$.

\section{Results}

\section{Serum glucose and insulin levels}

Hyperinsulinemic euglycemic clamp studies were performed in awake catheterized rats. Serum glucose and insulin levels under basal conditions and during steady state hyperinsulinemia are shown in Table I for each of the study protocols. Serum glucose was clamped near the basal glucose concentration throughout all clamp studies and the mean value did not differ significantly among the protocols $(P=\mathrm{NS})$. Steady state hyperinsulinemia $(\sim 300 \mu \mathrm{U} / \mathrm{ml})$ achieved as a result of the insulin infusions was also similar among study groups.

\section{Glucose turnover}

Study I. After the induction of hyperinsulinemia, glucose infusion rates required to maintain euglycemia increased rapidly and reached steady state at $\sim 60 \mathrm{~min}$. With the initiation of the glucosamine administration, glucose infusion rates decreased within 20-30 min (Fig. 1). The fall in glucose infusion in response to glucosamine was dose dependent and resulted in lower isotopically determined steady state glucose disappearance rates at all doses (Fig. 2). The maximal reduction in the glucose infusion rate under these conditions was $32.6 \pm 1.5 \%$ ( $P$ $<0.001$ ) and occurred at glucosamine infusion rate at or above $3.5 \mathrm{mg} / \mathrm{kg} / \mathrm{min}$. Even at doses as low as $0.1 \mathrm{mg} / \mathrm{kg} / \mathrm{min}$, glucosamine caused a $17.2 \pm 7.3 \%$ decline in glucose disposal rates $(P$ $<0.01)$. Compared with a glucosamine dose of $0.1 \mathrm{mg} / \mathrm{kg} /$ $\mathrm{min}$, the reduction in glucose disposal achieved at a glucosamine infusion rate of $3.5 \mathrm{mg} / \mathrm{kg} / \mathrm{min}$ was significantly greater $(P$ $<0.05$ ); however, there was no overall difference in glucose disposal rates among the various glucosamine doses $(P=$ NS, ANOVA). The apparent $\mathrm{ED}_{50}$ for glucosamine's effect to reduce glucose infusion rates was estimated to be at or below 0.1 $\mathrm{mg} / \mathrm{kg} / \mathrm{min}$ (Fig. 2).

Isotopically determined glucose disposal rates were not significantly different from the exogenous glucose infusion rates because rates of HGO were completely suppressed (not differ- 


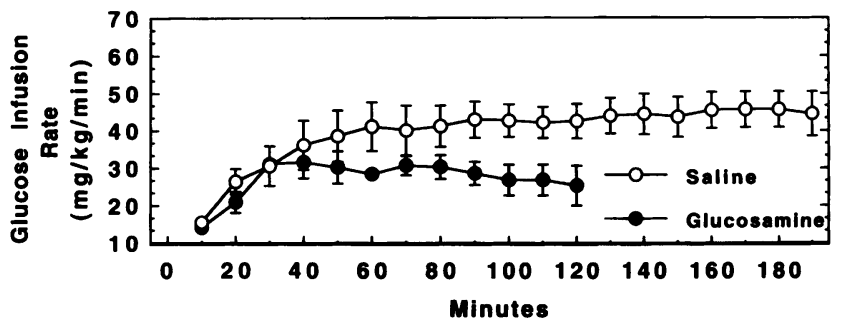

Figure 3. Control studies for effects of time course on glucose infusion rates during euglycemic hyperinsulinemic clamps. Prolonged 200-min euglycemic hyperinsulinemic clamp performed in five rats demonstrating no effect of time on glucose infusion rate during saline control studies is shown in open circles. The effect of glucosamine infusion ( $3.5 \mathrm{mg} / \mathrm{kg} / \mathrm{min}$ ) begun concomitantly with insulin (time 0 ) on glucose infusion rate in a separate group of five rats is shown in closed circles. Data are mean \pm SEM.

ent from zero) by the degree of hyperinsulinemia achieved during the clamp.

Study II. Insulin infusion without glucosamine led to a rapid increase in the glucose infusion rate and reached steady state within $60 \mathrm{~min}$. Importantly, there was no decline in glucose disposal rates when the clamp was prolonged past $120 \mathrm{~min}$. Therefore, the fall in glucose uptake observed in study I with glucosamine infusion cannot be attributed to a time course effect.

With concomitant infusion of glucosamine, steady state insulin action was reached at $\sim 40 \mathrm{~min}$ and was reduced by $38 \%$ compared with insulin alone from $42.1 \pm 4.2$ to $26.3 \pm 4.5 \mathrm{mg} /$ $\mathrm{kg} / \mathrm{min}(P<0.001)$, respectively (Fig. 3$)$. Thus, whether administered at the onset of the insulin infusion (study II) or after a period of maximal insulin stimulation (study I), glucosamine produced a comparable $33-38 \%$ reduction in rates of in vivo insulin-mediated glucose uptake.

Study III. To examine the specificity of glucosamine-induced insulin resistance, we measured rates of glucose disposal achieved during euglycemic hyperinsulinemic clamps with coinfusion of saline $(n=5)$, or mannosamine and galactosamine at rates of $3.5 \mathrm{mg} / \mathrm{kg} / \mathrm{min}$ in separate groups of rats $(n$ $=4$ each), and compared these rates to those achieved during coinfusion of glucosamine. During saline infusion, the maximally stimulated glucose disposal rate was $42.1 \pm 4.2 \mathrm{mg} / \mathrm{kg}$ / min. Compared with saline infusion, during glucosamine infusion the glucose disposal rate was reduced by $29 \%$ to $29.8 \pm 3.6$ $\mathrm{mg} / \mathrm{kg} / \mathrm{min}(P<0.001)$. In groups infused with mannosamine and galactosamine, glucose disposal rates were $36.1 \pm 2.8$ and $43.2 \pm 4.9 \mathrm{mg} / \mathrm{kg} / \mathrm{min}(P=\mathrm{NS}$ versus saline, respectively, ANOVA), respectively, indicating that mannosamine and galactosamine had no effect on insulin responsiveness when compared with the saline-infused group (Fig. 4).

\section{In vitro studies in skeletal muscle}

Such pronounced effects on total body glucose uptake during the clamp studies would necessarily have involved glucose uptake into skeletal muscle. To assess mechanisms of insulin resistance, we examined whether glucosamine altered translocation and subcellular distribution of GLUT 4 glucose transporter proteins. Hindlimb muscle was obtained from rats under basal conditions and during steady state hyperinsulinemia with and with-

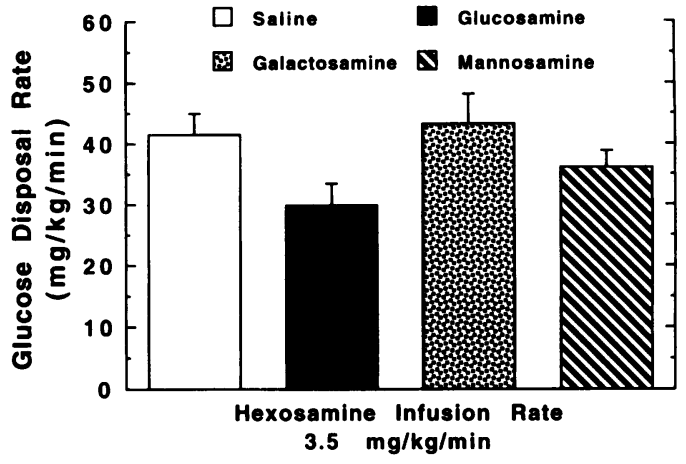

Figure 4. Glucose disposal rates in separate groups of animals ( $n=4$ each) studied during euglycemic hyperinsulinemia with coinfusion of glucosamine, mannosamine, and galactosamine compared with the saline control group in study II. Hexosamine infusion was begun at the same time as the insulin infusion (time 0). Data are mean \pm SEM.

out glucosamine infusion, and was subjected to a membrane subfractionation protocol using differential centrifugation and discontinuous sucrose density gradients. GLUT 4 levels in the various subcellular fractions were quantitated by polyacrylamide gel electrophoresis of membrane proteins followed by immunoblot analysis. Fig. 5 depicts a representative immunoblot. In this particular experiment, muscle membrane fractions were prepared from insulin-infused rats with and without coinfusion of glucosamine. Glucosamine did not alter the apparent molecular mass of GLUT 4 (migrating as a complex band centered at $46 \mathrm{kD}$ ) nor the amount of GLUT 4 in total muscle membranes (Fig. 5, lanes 5 and 6), but did reduce GLUT 4 in membranes collected at the interface of the $25 \%$ sucrose density gradient concomitant with an increase in the membrane fraction at the $35 \%$ sucrose interface.

GLUT 4 was quantitated in additional experiments performed in basal and insulin-infused rats, and mean data are shown in Fig. 6. Compared with basal membranes, hyperinsulinemia increased GLUT 4 by 2.6 -fold in membranes within the $25 \%$ sucrose fraction $(P<0.01)$ together with a $37 \%$ decrease

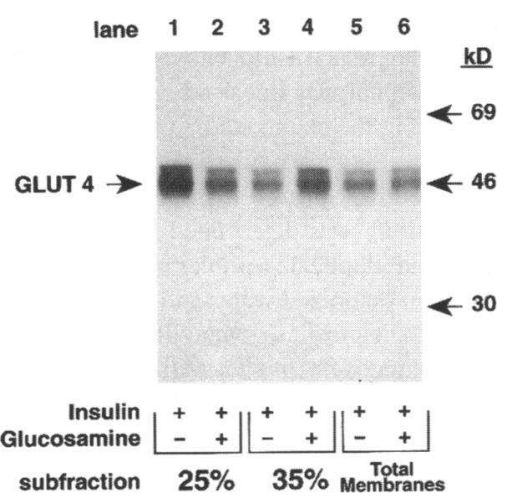

Figure 5. Immunological detection of GLUT 4 glucose transporters in rat muscle membrane subfractions. Awake catheterized rats were infused with insulin $(12 \mathrm{mU} / \mathrm{kg} /$ $\min )$ for $4 \mathrm{~h}$ with or without glucosamine ( 3.5 $\mathrm{mg} / \mathrm{kg} / \mathrm{min}$ ) for the final 2 h. Hindlimb muscle was then processed to obtain membrane subfractions by differential centrifugation and dis-

continuous sucrose density gradient separation. Membranes collecting at 25 and $35 \%$ sucrose gradients and total postnuclear membranes were studied. Equal amounts of membrane protein $(30 \mu \mathrm{g})$ were resolved by SDS-PAGE, transferred to nitrocellulose filters, and reacted with antibodies specific for GLUT 4 followed by ${ }^{125}$ I-protein A. Cross-reacting proteins were detected by autoradiography. 


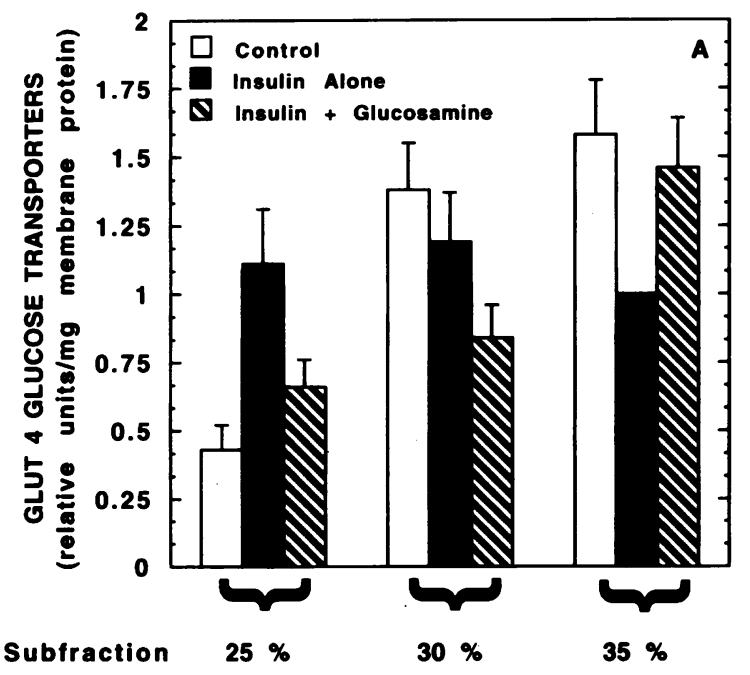

in the $35 \%$ sucrose fraction $(P<0.02)$, but did not affect $(P$ $=$ NS) GLUT 4 levels in the $30 \%$ sucrose fraction (Fig. $6 A$ ). These data are consistent with previous studies and have been interpreted to reflect insulin-mediated translocation of GLUT 4 from intracellular membranes in the $35 \%$ sucrose fraction to sarcolemma retrieved in the $25 \%$ sucrose fraction $(28,29)$. Importantly, coinfusion of glucosamine had marked effects on the subcellular distribution of GLUT 4. In maximally insulinstimulated animals, glucosamine decreased GLUT 4 levels in the $25 \%$ sucrose fraction by $41 \%(P<0.01$; glucosamine plus insulin versus insulin alone). In addition, glucosamine completely blocked insulin-induced loss of GLUT 4 in the $35 \%$ sucrose fraction since GLUT 4 levels were similar in comparing basal and glucosamine plus insulin-infused animals $(P=$ NS $)$. Thus, glucosamine prevented translocation of GLUT 4 from the 35 to the $25 \%$ sucrose gradient membrane subfraction.

Glucosamine was also observed to decrease GLUT 4 in the $30 \%$ sucrose fraction $(29 \% ; P<0.05)$, a membrane subfraction which was not affected by insulin per se. To more carefully examine this cellular compartment, membranes at the $30 \%$ sucrose interface were collected, washed, and subjected to a second sucrose density separation which used 27 as well as $30 \%$ sucrose density gradients. Mean GLUT 4 levels in these latter membrane subfractions are shown in Fig. $6 \mathrm{~B}$. It was clear that insulin mobilized GLUT 4 away from the $30(P<0.05)$ but not the $27 \%$ ( $P=\mathrm{NS}$ ) fraction (i.e., compared with basal), and that glucosamine coinfusion decreased GLUT 4 only in the $27 \%$ sucrose fraction $(P<0.05)$ without affecting levels $(P$ $=\mathrm{NS}$ ) in the $30 \%$ fraction. Therefore, the original $30 \%$ subfraction resulting from the first sucrose density gradient separation was comprised of two functionally distinct membrane species; when subjected to a second gradient separation, membranes were recovered at $27 \%$ sucrose which were sensitive to glucosamine but not insulin, and the remaining membranes collecting at $30 \%$ sucrose were sensitive to insulin but not to glucosamine.

We performed additional experiments to ensure that altered amounts of GLUT 4 in membrane subfractions were not due to
Figure 6. Effects of insulin and glucosamine on GLUT 4 in muscle membrane subfractions. Rats were infused for $4 \mathrm{~h}$ with either saline, insulin, or insulin plus glucosamine, and hindlimb muscle was processed to obtain membrane subfractions, as described for Fig. 5. Membranes collecting at 25,30 , and $35 \%$ sucrose gradients were analyzed in $A$; the $30 \%$ sucrose fraction was also subjected to a second sucrose density gradient separation, and membranes collecting at $27[27 \%(a)]$ and $30 \%$ [30\% $(a)]$ sucrose gradients were analyzed in $B$. GLUT 4 was detected in each membrane subfraction by immunoblot analysis as shown in Fig. 5 and quantitated by excising the GLUT 4 band and counting radioactivity. Data are mean \pm SEM for seven experiments. effects of glucosamine on GLUT 4 recovery, membrane subfractionation, or overall muscle GLUT 4 expression. Muscle content of GLUT 4 was quantitated by immunoblot analyses of total postnuclear membranes and was not affected by insulin or glucosamine infusions as delineated in Table IV $(P=$ NS $)$. We also expressed GLUT 4 levels in membrane subfractions as a fraction of GLUT 4 in total membrane fractions from individual experiments to provide a measure of relative GLUT 4 recovery (Table IV). When expressed in this way, the data were similar to that in Fig. 5 where GLUT 4 levels were normalized per milligram of membrane protein. We consistently observed that insulin translocated GLUT 4 from membranes in the 35 to the $25 \%$ sucrose fractions with no effects on the $30 \%$ fraction, that this effect of insulin was blocked by glucosamine, and that the $30 \%$ fraction was complex, containing membrane species which were differentially sensitive to effects of glucosamine and insulin. In other experiments we measured three sarcolemmal marker proteins in all membrane fractions, namely, $\mathrm{Na}^{+} / \mathrm{K}^{+}$ATPase activity, 5'-nucleotidase activity, and immunoreactive phospholemman (Table $\mathrm{V}$ ). $\mathrm{Na}^{+} / \mathrm{K}^{+}$ATPase specific activity was greatest in the $25 \%$ sucrose fraction, and enrichment of 5'-nucleotidase was highest in the 25,27 , and $30 \%$ fractions with a lower value in the $35 \%$ fraction. Phospholemman, a chloride channel which is densely distributed across all domains of sarcolemma $(32,33)$, was most enriched in 25,27 , and $35 \%$ sucrose fractions. Importantly, coinfusion of glucosamine compared with insulin alone did not affect enrichment of either 5'-nucleotidase, $\mathrm{Na}^{+} / \mathrm{K}^{+}$ATPase, or phospholemman in any of the membrane subfractions $(P=\mathrm{NS})$, and, therefore, glucosamine's ability to block insulin-mediated translocation of GLUT 4 could not be ascribed to changes in membrane subfractionation.

\section{Discussion}

This study was designed to address the following hypotheses: (a) circulating glucosamine causes insulin resistance in vivo; 
Table IV. Effect of Glucosamine on the Subcellular Distribution of GLUT 4 Glucose Transporters in Rat Skeletal Muscle during Hyperinsulinemic Euglycemic Clamps: GLUT 4 in Subfractions Normalized for Total Muscle GLUT 4 Content

\begin{tabular}{lcccccc}
\hline & & \multicolumn{4}{c}{ GLUT 4 in membrane subfraction/GLUंT 4 in total membranes (relative recovery of GLUT 4)* } \\
\cline { 3 - 6 } Rat treatment subgroup & Total membrane GLUT 4 & $25 \%$ & $30 \%$ & $35 \%$ & $27 \% \mathrm{a}$ & $30 \% \mathrm{a}$ \\
\hline & (relative units/mg protein) & & & & \\
Basal & $1.14 \pm 0.12$ & $0.61 \pm 0.12$ & $1.12 \pm 0.22$ & $1.44 \pm 0.08$ & $1.41 \pm 0.25$ & $1.31 \pm 0.18$ \\
Insulin & $0.98 \pm 0.13$ & $1.11 \pm 0.22$ & $1.18 \pm 0.20$ & 1.00 & $1.50 \pm 0.19$ & $1.07 \pm 0.24$ \\
Insulin + glucosamine & $0.94 \pm 0.15$ & $0.69 \pm 0.13$ & $0.87 \pm 0.11$ & $1.52 \pm 0.13$ & $1.11 \pm 0.22$ & $1.03 \pm 0.20$ \\
& & & & & & \\
\hline
\end{tabular}

* Values are relative units; the ratio in the $35 \%$ sucrose density gradient fraction from rats infused with insulin alone was assigned a value of 1.00 . Awake catheterized rats were infused for $4 \mathrm{~h}$ with either saline (Basal), $12 \mathrm{mU} / \mathrm{m}^{2} / \mathrm{min}$ insulin (Insulin), or insulin with coinfusion of $3.5 \mathrm{mg} / \mathrm{kg}$ / min glucosamine for the final $2 \mathrm{~h}$ (Insulin + glucosamine). Hindlimb muscle was then processed to obtain membrane subfractions by differential centrifugation and sucrose density gradient separation. Membranes collecting at the interface of the 25,30 , and $35 \%$ discontinuous sucrose density gradients were retrieved for analysis; membranes at the interface of the $30 \%$ sucrose interface were also subjected to a second discontinuous sucrose density gradient separation and membranes were collected atop the $27 \%(27 \% a)$ and $30 \%(30 \% a)$ gradients. Equal amounts of membrane protein were subjected to SDS-PAGE and immunoblot analysis for measurement of GLUT 4. To obtain a measure of GLUT 4 recovery, GLUT 4 levels in the membrane subfractions (Fig. 5) were normalized for the amount of GLUT 4 in total muscle membranes from individual rats in each experimental subgroup. Total membrane GLUT 4 was measured in the high-speed pellet ( $190,000 \mathrm{~g}$ for $60 \mathrm{~min})$ before application of the resuspended pellet to the first sucrose density gradient. Data represent mean \pm SEM of seven experiments.

and $(b)$ glucosamine causes insulin resistance by inducing a defect in glucose transporter GLUT 4 translocation in skeletal muscle. The in vivo data indicate that glucosamine causes a marked decrease in rates of insulin-mediated glucose uptake into peripheral tissues. The in vitro data indicate that glucos- amine causes an impairment in insulin's ability to cause translocation of GLUT 4 glucose transporters in skeletal muscle. Taken together, the data suggest that glucosamine-induced insulin resistance is secondary (at least in part) to a defect in glucose transporter translocation.

Table V. Sarcolemmal Marker Proteins in Subcellular Membrane Fractions

\begin{tabular}{|c|c|c|c|c|}
\hline Sucrose gradient fraction & Rat treatment subgroup & 5 '-nucleotidase activity* & Immunoreactive phospholemman* & $\mathrm{Na}^{+} / \mathrm{K}^{+}$ATPase activity \\
\hline & & \multicolumn{2}{|c|}{ fold increase above value in total membranes } & $\mathrm{nmol} / \mathrm{mg} / \mathrm{min}$ \\
\hline & Insulin & $2.2 \pm 0.4$ & $1.9 \pm 0.2$ & $3.2 \pm 0.5$ \\
\hline \multicolumn{5}{|l|}{$25 \%$} \\
\hline & Insulin + glucosamine & $2.4 \pm 0.6$ & $2.1 \pm 0.1$ & $3.4 \pm 0.5$ \\
\hline & Insulin & $3.3 \pm 0.4$ & $1.8 \pm 0.2$ & $2.8 \pm 0.7$ \\
\hline \multicolumn{5}{|l|}{$30 \%$} \\
\hline & Insulin + glucosamine & $3.5 \pm 0.3$ & $1.9 \pm 0.6$ & $2.9 \pm 0.7$ \\
\hline & Insulin & $1.6 \pm 0.1$ & $4.2 \pm 0.6$ & $1.2 \pm 0.2$ \\
\hline \multicolumn{5}{|l|}{$35 \%$} \\
\hline & Insulin + glucosamine & $1.5 \pm 0.1$ & $3.9 \pm 0.6$ & $1.2 \pm 0.2$ \\
\hline & Insulin & $5.3 \pm 1.1$ & $2.9 \pm 0.3$ & $2.9 \pm 0.7$ \\
\hline \multicolumn{5}{|l|}{$27 \% a$} \\
\hline & Insulin + glucosamine & $5.0 \pm 0.9$ & $3.7 \pm 1.5$ & $2.8 \pm 0.7$ \\
\hline & Insulin & $4.0 \pm 0.5$ & $1.2 \pm 0.2$ & $2.0 \pm 0.1$ \\
\hline \multicolumn{5}{|l|}{$30 \% a$} \\
\hline & Insulin + glucosamine & $4.0 \pm 0.8$ & $1.6 \pm 0.7$ & $2.2 \pm 0.2$ \\
\hline
\end{tabular}

* In total membranes from insulin- and insulin + glucosamine-infused rats, the respective mean \pm SEM values for 5 -nucleotidase activity $(1.3 \pm 0.3$ and $1.4 \pm 0.3 \mu \mathrm{mol} / \mathrm{mg}$ protein $/ \mathrm{h}), \mathrm{Na}^{+} / \mathrm{K}^{+}$ATPase activity $(1.0 \pm 0.2$ and $0.9 \pm 0.3 \mathrm{nmol} / \mathrm{mg}$ protein $/ \mathrm{min})$, and immunoreactive phospholemman $(1.0 \pm 0.1$ and $1.0 \pm 0.2$ relative units $/ \mathrm{mg}$ membrane protein) were similar $(P=\mathrm{NS})$. Hindlimb skeletal muscle was obtained from rats infused for $4 \mathrm{~h}$ with either insulin or insulin + glucosamine, and processed to obtain membrane subfractions by differential centrifugation and sucrose density gradient separation. Membranes collecting at 25, 30, and 35\% discontinuous sucrose density gradients were retrieved for analysis; membranes at the $30 \%$ sucrose interface were also subjected to a second sucrose density gradient separation and membranes were collected at the $27 \%(27 \% a)$ and 30\% (30\%a) sucrose interfaces. 5'-nucleotidase activity and phospholemman levels represent the fold increase of values over those observed in total muscle membranes. Total membrane parameters were measured in the high-speed pellet $(190,000 \mathrm{~g}$ for $60 \mathrm{~min})$ before application to the resuspended pellet to the first sucrose density gradient. Oubain-inhibitable $\mathrm{Na}^{+} \mathrm{K}^{+}$ATPase data are expressed as specific activity per milligram of membrane protein. Data are mean \pm SEM of three to four experiments. 
Glucose-induced desensitization of insulin action is mediated via flux through the hexosamine biosynthetic pathway (26). Glucosamine is a metabolic product of the hexosamine pathway and is even more potent than glucose in inducing insulin resistance in isolated adipocytes $(18,23)$. We reasoned that glucosamine infused in vivo would also result in insulin desensitization. Our data indicate that circulating glucosamine can induce marked insulin resistance. Indeed, we found that glucosamine induced a $30-40 \%$ reduction in rates of insulinstimulated glucose uptake, whether administered at the onset of the insulin infusion or after insulin action was allowed to be fully expressed. Moreover, the time course of glucosamineinduced insulin resistance indicates that the process occurs relatively rapidly (within $20-30 \mathrm{~min}$ ). The effect of glucosamine to decrease insulin-mediated glucose uptake appears to be dose dependent with an apparent $\mathrm{ED}_{50}$ of $\sim 0.1 \mathrm{mg} / \mathrm{kg} / \mathrm{min}$. Because we studied relatively small groups of rats, the exact $\mathrm{ED}_{50}$ cannot be confidently determined; however, it is clear that, even at infusion rates as low as $0.5 \mathrm{mg} / \mathrm{kg} / \mathrm{min}$ (a rate $\sim 1 / 70$ th of the overall insulin-stimulated glucose turnover rate on a molar basis), glucosamine can cause significant reductions in insulinmediated glucose uptake. Thus, glucosamine is a highly potent, rapid, and dose-dependent inducer of in vivo insulin resistance. Finally, because other hexosamines such as mannosamine and galactosamine had no effect to alter insulin-mediated glucose uptake, it is apparent that glucosamine's action to cause insulin desensitization in vivo is quite specific, thus recapitulating the previous in vitro data obtained in isolated adipocytes (18).

Because the insulin infusion rate used during the euglycemic clamps is maximally effective, it is clear that glucosamine causes a marked reduction in insulin responsiveness. Reductions in insulin responsiveness suggest that glucosamine caused a defect in a rate-limiting step of insulin-stimulated glucose uptake. Our data indicating a defect at the level of the glucose transport system are consistent with this notion. We did not examine the effect of glucosamine at lower insulin doses; however, it highly likely that glucosamine's antagonistic effects on rate-limiting steps in glucose uptake would also be manifest as a decrease in insulin sensitivity (i.e., insulin action at submaximally effective insulin concentrations). Furthermore, the magnitude of in vivo effects indicates that glucosamine-induced insulin resistance affects skeletal muscle which accounts for the bulk of glucose disposal.

With regard to underlying mechanisms, our data suggest that glucosamine induces insulin resistance by impairing insulinmediated translocation of intracellular GLUT 4 glucose transporters to sarcolemma. Insulin stimulation led to an approximately twofold increase in GLUT 4 in the $25 \%$ sucrose density gradient fraction concomitant with a $\sim 40 \%$ decrease in the $35 \%$ sucrose fraction. These observations agree very well with those from previous studies using a similar subfractionation protocol in rat muscle $(28,29,39)$. The data have been interpreted to represent translocation of GLUT 4 from internal membranes in the $35 \%$ fraction to sarcolemma in the $25 \%$ fraction based on enrichment of marker enzyme activities in these subfractions $(28,39,40)$ as well as quantitative correlation between the GLUT 4 increment in the $25 \%$ sucrose fraction and the extent of glucose transport activity stimulation (29). An important finding in the current studies is that infusion of glucosamine effectively blocked insulin's ability to both augment
GLUT 4 in the $25 \%$ sucrose fraction and decrease GLUT 4 in the $35 \%$ sucrose fraction.

To monitor membrane subfractionation, we have studied $\mathrm{Na}^{+} / \mathrm{K}^{+}$ATPase activity which was enriched in the $25 \%$ sucrose fraction, 5'-nucleotidase which was enriched in the 25$30 \%$ sucrose fractions, as well as phospholemman, a recently cloned chloride channel densely distributed across all domains of sarcolemma $(32,33)$. Previous muscle subfractionation studies $(28,29,39,40)$ showing sarcolemmal enrichment in the $25 \%$ sucrose fraction relied heavily on $\mathrm{Na}^{+} / \mathrm{K}^{+}$ATPase which, in epithelial cells, is localized to specific plasma membrane domains juxtaposed to ankyrin/fodrin complexes and other elements of membrane skeleton (41). Phospholemman is an excellent sarcolemmal marker in myocardium where it is densely distributed across all cell surface domains but has not been rigorously localized in skeletal muscle. However, the fact that $\mathrm{Na}^{+} / \mathrm{K}^{+}$ATPase, $5^{\prime}$-nucleotidase, and phospholemman are detected in all sucrose fractions may indicate that sarcolemmal domains vary widely in density. By inference, therefore, translocation of GLUT 4 to sarcolemma is not uniform but appears to involve specific domains which are enriched in the $25 \%$ sucrose fraction. The important consideration is that glucosamine did not alter recovery of marker proteins in our subfractions and that the ability to prevent translocation cannot be ascribed to nonspecific effects on membrane subfractionation per se.

The ability of glucosamine to impair GLUT 4 translocation could result from either: $(a)$ defects in insulin signal transduction; or $(b)$ defects intrinsic to the glucose transport effector system involving alterations in the trafficking/translocation of GLUT 4-containing vesicles. While either of these explanations is possible, we favor the latter mechanism for four reasons. The first reason is based on experimental observations in the $30 \%$ sucrose membrane subfraction. Current data and previous studies (28) show no effect of insulin in altering levels of GLUT 4 in this cellular compartment. However, we discovered that the $30 \%$ sucrose fraction was complex and comprised of functionally distinct membrane subpopulations differentially sensitive to the effects of glucosamine and insulin. We subjected these membranes to a second sucrose density gradient separation to resolve $27 \%$ as well as $30 \%$ sucrose subfractions. GLUT 4 in the $27 \%$ sucrose fraction was decreased by glucosamine but not affected by insulin, whereas GLUT 4 in the $30 \%$ sucrose fraction was diminished by insulin but resistant to effects of glucosamine. Thus, within this compartment, glucosamine altered subcellular localization of an insulin-insensitive pool of GLUT 4. If glucosamine can alter membrane localization of a GLUT 4 pool not affected by insulin signal transduction, it is reasonable to hypothesize that glucosamine could also affect localization of the insulin-responsive GLUT 4 pool as a mechanism responsible for impaired translocation.

The second reason for favoring a defect intrinsic to the glucose transporter system pertains to our recent observations in adipocytes from women with gestational diabetes mellitus who are characterized by endogenous hyperglycemia and hyperinsulinemia. In these patients, insulin resistance is associated with abnormal accumulation of GLUT 4 in vesicles cofractionating with both high-density microsomes and plasma membranes in basal cells, combined with the absence of GLUT 4 translocation in response to insulin (42). Thus, GLUT 4 appears to be relegated to a cellular compartment from which insulin cannot recruit transporters to the cell surface consistent with 
abnormalities in cellular GLUT 4 targeting and/or trafficking. These defects arose under in vivo conditions which would tend to promote high flux through the hexosamine pathway. The third reason is based on experiments in isolated rat adipocytes. In these cells, glucose- (or glucosamine-) induced insulin resistance is dependent upon metabolic flux through the hexosamine pathway (23) and is the consequence of impaired translocation of glucose transporters to the cell surface (18). The translocation defect arises in the absence of any abnormalities in insulin receptor tyrosine kinase activity and IRS-1 phosphorylation (20), and importantly is not associated with impaired stimulation of other insulin bioeffects including protein synthesis, glycogen synthase, glucose oxidation, and glucose incorporation into lipid (21). Finally, Buse et al. (43) have found recently that glucosamine does not inhibit insulin signal transduction and stimulation of glucose transport in rat-1 fibroblasts which exclusively express GLUT 1 . Since GLUT 1 and GLUT 4 are differentially targeted to different intracellular vesicles (2), glucosamine may specifically impair translocation of GLUT 4containing vesicles. For all above-mentioned reasons, we postulate that glucosamine induces insulin resistance via defects in trafficking/targeting of GLUT 4 vesicles which interfere with their recruitment to the cell surface.

The molecular basis of glucosamine-induced insulin resistance could involve alterations in gene transcription or posttranslational glycosylation of critical proteins which regulate GLUT 4 translocation. The effects of high glucose or glucosamine, to alter gene expression of GFAT (24) and pyruvate kinase (44) in adipocytes, and induction of TGF- $\alpha$ in vascular smooth muscle cells (45), are mediated by products of the hexosamine biosynthetic pathway. An important metabolic byproduct of this pathway is UDP- $N$-acetylglucosamine which is a critical reactant for both $\mathrm{O}$-linked and $\mathrm{N}$-linked glycosylation. Transcription factors, including $\mathrm{Sp} 1$, can be rapidly modified by O-linked glycosylation and this affects binding affinity at consensus sites in gene promoter regions $(46,47)$. Interestingly, pyruvate kinase and TGF- $\alpha$ genes possess Sp1 binding sites in 5 '-flanking DNA (45). Posttranslational glycosylation could similarly modify the activity of proteins involved in GLUT 4 vesicle trafficking or docking to plasma membrane. While these potential mechanisms remain speculative, our studies establish an animal model of skeletal muscle insulin resistance for further elucidation of underlying defects.

Our observations have important implications regarding the pathogenesis of insulin resistance in diabetes mellitus. In NIDDM, insulin resistance can be partially reversed by treatment modalities which lower ambient hyperglycemia (5-11, 48 ). Therefore, it appears that insulin resistance is multicomponential and comprised of reversible and nonreversible biochemical defects. In IDDM, euglycemia appears to lead to complete reversal of insulin resistance $(12,13)$. The current data implicate the hexosamine pathway in glucose-induced insulin resistance in skeletal muscle. This mechanism is potentially relevant to diabetic patients. Certainly, defective translocation as a mechanism of insulin resistance is compatible with the observation that GLUT 4 expression in skeletal muscle is normal in both NIDDM (42) and IDDM (49). These studies establish a new animal model of insulin resistance which specifically allows for additional studies defining the role of the hexosamine pathway and molecular mechanisms mediating glucose toxicity.

\section{Acknowledgments}

The authors wish to thank Shirley Kendrick and Ginger Brechtel-Hook for their invaluable assistance in the preparation of the manuscript.

This work was supported by research grants DK-20542, DK-38765, and DK-42469 from the National Institutes of Health, the Merit Review program of the Department of Veterans Affairs, and the American Heart Association.

\section{References}

1. DeFronzo, R. A., R. C. Bonadonna, and E. Ferrannini. 1992. Pathogenesis of NIDDM-A balanced overview. Diabetes Care. 15:318-368.

2. Garvey, W. T., and M. Birnbaum. 1993. Cellular insulin action and insulin resistance. In Clinical Endocrinology and Metabolism. E. Ferrannini, editor. Bailliere Tindall, London. 785-873.

3. Unger, R. H., and S. Grundy. 1985. Hyperglycaemia as an inducer as well as a consequence of impaired islet cell function and insulin resistance: implications for the management of diabetes. Diabetologia. 28:119-121.

4. Rossetti, L., A. Giaccari, and R. A. DeFronzo. 1990. Glucose toxicity. Diabetes. 3:610-630.

5. Henry, R. R., P. Wallace, and J. M. Olefsky. 1986. Effects of weight loss on mechanisms of hyperglycemia in obese non-insulin dependent diabetes mellitus. Diabetes. 35:990-998.

6. Kolterman, O. G., R. S. Gray, G. Shapiro, J. A. Scarlett, J. Griffin, and J. M. Olefsky. 1984. The acute and chronic effects of sulfonylurea therapy in type II diabetic subjects. Diabetes. 33:346-354.

7. Greenfield, M. S., L. Doberne, M. Rosenthal, B. Schultz, A. Widstrom, and G. M. Reaven. 1982. Effects of sulfonylurea treatment on in vivo insulin secretion and action in patients with non-insulin-dependent diabetes mellitus. Diabetes. 31:307-312.

8. Ginsberg, H., and E. J. Rayfield. 1981. Effect of insulin therapy on insulin resistance in type II diabetic subjects: evidence for heterogeneity. Diabetes. 30:739-745.

9. Garvey, W. T., J. M. Olefsky, J. Griffin, R. F. Hamman, and O. G. Kolterman. 1985. The effect of insulin treatment on insulin secretion and insulin action in type II diabetes mellitus. Diabetes. 34:222-234.

10. Scarlett, J., O. G. Kolterman, T. P. Ciaraldi, M. Kao, and J. O. Olefsky. 1983. Insulin treatment reverses the post receptor defect in adipocyte 3-O-methyl glucose transport in type II diabetes mellitus. J. Clin. Endocrinol. \& Metab. 56:1195-1201.

11. Friedman, J. E., L. Dohm, N. Leggett-Frazier, C. W. Elton, B. E. Tapscott, W. P. Pories, and J. F. Caro. 1992. Restoration of insulin responsiveness in skeletal muscle of morbidly obese patients after weight loss: effect on muscle glucose transport and glucose transporter GLUT 4. J. Clin. Invest. 89:701-705.

12. Revers, R. R., O. G. Kolterman, J. A. Scarlett, R. S. Gray, and J. M. Olefsky. 1984. Lack of in vivo insulin resistance in controlled insulin dependent type I diabetic patients. J. Clin. Endocrinol. \& Metab. 58:353-358.

13. Yki-Jarvinen, H., E. Helve, and V. A. Koivisto. 1987. Hyperglycemia decreases glucose uptake in type I diabetes. Diabetes. 36:892-896.

14. Karnielli, E., M. Armoni, P. Cohen, Y. Kanter, and R. Rafaeloff. 1987. Reversal of insulin resistance in diabetic rat adipocytes by insulin therapy: restoration of pool of glucose transporters and enhancement of glucose-transport activity. Diabetes. 36:925-931.

15. Rossetti, L., D. Smith, G. I. Schulman, D. Papachristou, and R. A. DeFronzo. 1987. Correction of hyperglycemia with phlorizin normalizes tissue sensitivity to insulin in diabetic rats. J. Clin. Invest. 87:1510-1515.

16. Kahn, B., G. I. Schulman, R. A. DeFronzo, S. W. Cushman, and L. Rossetti. 1991. Normalization of blood glucose in diabetic rats with phlorizin treatment reverses insulin-resistant glucose transport in adipose cells without restoring glucose transporter gene expression. J. Clin. Invest. 87:561-570.

17. Richter, E. A., B. F. Hansen, and S. A. Hansen. 1988. Glucose-induced insulin resistance of skeletal-muscle glucose transport and uptake. Biochem. J. 252:733-737.

18. Garvey, W. T., J. M. Olefsky, S. Matthaei, and S. Marshall. 1987. Glucose and insulin co-regulate the glucose transport system in primary cultured adipocytes: a new mechanism of insulin resistance. J. Biol. Chem. 262:189-197.

19. Garvey, W. T., T. P. Huecksteadt, F. B. Lima, and J. M. Birnbaum. 1989. Expression of a glucose transporter gene cloned from brain in cellular models of insulin resistance: dexamethasone decreases transporter mRNA in primary cultured adipocytes. Mol. Endocrinol. 3:1132-1141.

20. Lima, F. B., R. S. Thies, and W. T. Garvey. 1991. Glucose and insulin regulate insulin sensitivity in primary cultured adipocytes without affecting insulin receptor kinase activity. Endocrinology. 128:2415-2426.

21. Lima, F. B., S. Bao, and W. T. Garvey. 1994. Biological actions of insulin 
are differentially regulated by glucose and insulin in primary cultured adipocytes: chronic ability to increase glycogen synthase activity. Diabetes. 43:53-62.

22. Traxinger, R. R., and S. Marshall. 1989. Role of amino acids in modulating glucose induced desensitization of the glucose transport system. J. Biol. Chem. 264:20910-20916.

23. Marshall, S., V. Bacote, and R. R. Traxinger. 1991. Discovery of a metabolic pathway mediating glucose induced desensitization of the glucose transport system: role of hexosamine biosynthesis in the induction of insulin resistance. $J$. Biol. Chem. 266:4706-4712.

24. Traxinger, R. R., and S. Marshall. 1991. Coordinated regulation of glutamine: fructose-6-phosphate amidotransferase activity by insulin, glucose, and glutamine: role of hexosamine biosynthesis in enzyme regulation. J. Biol. Chem. 266:10148-10154.

25. Marshall, S., V. Bacote, and R. R. Traxinger. 1991. Complete inhibition of glucose-induced desensitization of the glucose transport system by inhibitors of mRNA synthesis: evidence for rapid turnover of glutamine: fructose-6-phosphate amidotransferase. J. Biol. Chem. 266:10155-10161.

26. Marshall, S., W. T. Garvey, and R. R. Traxinger. 1991. New insights into the metabolic regulation of insulin action and insulin resistance: role of glucose and amino acids. FASEB (Fed. Am. Soc. Exp. Biol.) J. 5:3031-3036.

27. Smith, D., L. Rossetti, E. Ferrannini, C. M. Johnson, C. Cobelli, G. Toffolo, L. D. Katz, and R. A. DeFronzo. 1987. In vivo glucose metabolism in the awake rat: tracer and insulin clamp studies. Metab. Clin. Exp. 36:1167-1174.

28. Klip, A., T. Ramlal, D. A. Young, and J. O. Holloszy. 1987. Insulininduced translocation of glucose transporters in rat hindlimb muscles. FEBS Lett. 224:224-230.

29. Klip, A., T. Ramlal, P. J. Bilan, G. D. Cartee, E. A. Gulve, and J. O. Holloszy. 1990. Recruitment of GLUT 4 glucose transporters by insulin in diabetic rat skeletal muscle. Biochem. Biophys. Res. Commun. 172:728-736.

30. Avruch, J., and D. F. H. Wallach. 1971. Preparation and properties of plasma membrane and endoplasmic reticulum fragments from isolated rat fat cells. Biochim. Biophys. Acta. 233:334-347.

31. Forbush, B. 1983. Assay of Na/K-ATPase in plasma membranes. Anal. Biochem. 128:159-163.

32. Palmer, C. J., B. T. Scott, and L. R. Jones. 1991. Purification and complete sequence determination of the major plasma membrane substrate for cAMPdependent protein kinase and protein kinase $\mathrm{C}$ in myocardium. J. Biol. Chem. 266:11126-11130.

33. Walaas, S. I., R. S. Horn, K. A. Albert, A. Adler, and O. Walaas. 1988. Phosphorylation of multiple sites in a 15000 dalton proteolipid from rat skeletal muscle sarcolemma, catalyzed by adenosine $3^{\prime}, 5^{\prime}$-monophosphate dependent and calcium/phospholipid-dependent protein kinases. Biochim. Biophys. Acta. 968: 127-137.

34. Laemmli, U. K. 1970. Cleavage of structural proteins during assembly of the head of bacteriophage $\mathrm{T}_{4}$. Nature (Lond.). 227:680-685.

35. Towbin, H., T. Staehelin, and J. Gordon. 1979. Electrophoretic transfer of proteins from polyacrylamide gels to nitrocellulose sheets: procedure and some applications. Proc. Natl. Acad. Sci. USA. 76:4350-4354.

36. Garvey, W. T., L. Maianu, J. Hancock, A. M. Golichowski, and A. D.
Baron. 1992. Gene expression of GLUT 4 in skeletal muscle from insulin-resistant patients with obesity, IGT, GDM and NIDDM. Diabetes. 41:465-475.

37. James, D. E., M. Strube, and M. Meuckler. 1989. Molecular cloning and characterization of an insulin-regulatable glucose transporter. Nature (Lond.). 338:83-87.

38. Bradford, M. 1976. A rapid and sensitive method for the quantitation of microgram quantities of protein utilizing the principal protein-dye bonding. Anal. Biochem. 72:248-254.

39. Hirshman, M. F., L. J. Goodyear, L. J. Wardzala, E. D. Horton, and E. S. Horton. 1990. Identification of an intracellular pool of glucose transporters from basal and insulin-stimulated rat skeletal muscle. J. Biol. Chem. 265:987-991.

40. Sternlicht, E., R. J. Barnard, and G. K. Grimditch. 1988. Mechanisms of insulin action on glucose transport in rat skeletal muscle. Am. J. Physiol. 254:E633-E638.

41. Welson, W. J. 1993. Linkage of plasma membrane proteins with the membrane skeleton: insights into functions in polarized epithelial cells. In Molecular Mechanisms of Membrane Traffic. D. J. Morre, K. E. Howell, and J. J. M. Bergeron, editors. Springer-Verlag, Berlin. 273-283.

42. Garvey, W. T., L. Maianu, J.-H. Zhu, J. A. Hancock, and A. M. Golichowski. 1993. Multiple defects in the adipocyte glucose transport system cause cellula insulin resistance in gestational diabetes: heterogeneity in the number and a novel abnormality in subcellular localization of GLUT 4 glucose transporters. Diabetes. 42:1773-1785.

43. Robinson, K. A., D. A. Sens, and M. G. Buse. 1993. Pre-exposure to glucosamine induces insulin resistance of glucose transport and glycogen synthesis in isolated rat skeletal muscles: study of mechanisms in muscle and in rat-1 fibroblasts overexpressing the human insulin receptor. Diabetes. 42:1339-1346.

44. Traxinger, R. R., and S. Marshall. 1992. Insulin regulation of pyruvate kinase activity in isolated adipocytes: crucial role of glucose and the hexosamine biosynthesis pathway in the expression of insulin action. J. Biol. Chem. 267:97189723.

45. Daniels, M. C., P. Kansal, T. M. Smith, A. J. Paterson, J. E. Kudlow, and D. A. McClain. 1993. Glucose regulation of transforming growth factor-alpha expression is mediated by products of the hexosamine biosynthesis pathway. Mol. Endocrinol. 7:1041-1048.

46. Holt, G. D., and G. W. Hart. 1986. The subcellular distribution of terminal $\mathrm{N}$-acetylglucosamine moieties: localization of a novel protein saccharide linkage, O-linked Glc.NAc. J. Biol. Chem. 261:8049-8057.

47. Jackson, S. P., and R. Tijan. 1988. O-glycosylation of eukaryotic transcription factors: implications for mechanisms of transcriptional regulation. Cell. 55:125-133.

48. Baron, A. D., M. Laakso, G. Brechtel, and S. V. Edelman. 1991. Reduced capacity and affinity of skeletal muscle for insulin mediated glucose uptake in non-insulin-dependent diabetic subjects. Effects of insulin therapy. J. Clin. Invest. 87:1186-1194.

49. Yki-Jarvinen, H., H. Vuorinen-Markkola, L. Koranyi, R. Bourey, K. Tordjman, M. Mueckler, A. M. Permutt, and V. A. Koivisto. 1992. Defect in insulin action on expression of the muscle/adipose tissue glucose transporter gene in skeletal muscle of type I diabetic patients. J. Clin. Endocrinol. \& Metab. 75:795799. 